\title{
A regional study of presentation and outcome of hypertrophic cardiomyopathy in infants
}

\author{
J R Skinner, A Manzoor, A M Hayes, H S Joffe, R P Martin
}

\begin{abstract}
Objective-To describe regional incidence, presentation, and outcome of idiopathic (familial) and Noonan syndrome related infant hypertrophic cardiomyopathy (HCM) between 1969 and 1994.

Design-Case series.

Setting-Regional cardiac referral unit of the South West Region of England and south Wales, population approximately four million.
\end{abstract}

Patients-21 cases of idiopathic (or familial) HCM, and eight infants with Noonan syndrome.

Main outcome measures-Survival and persistence or resolution of symptoms or cardiac hypertrophy.

Results-Incidence: eight cases between 1969 and 1982 (idiopathic 6, Noonan 2), 21 cases between 1982 to 1994 (idiopathic 15, Noonan 6). Mode of presentation: cardiac failure, 17 (59\%); murmur, 9 (30\%); cyanosis, 2 (7\%); family history, $1(7 \%)$. Age at presentation: 0-7 days, 16 (55\%); 8 days-4 months, 9 (31\%); 5-12 months, 4 (14\%). Outcome: five deaths $(17 \%)$, all $<1$ year, all from progressive cardiac failure (idiopathic 3, Noonan 2). Four of these five had not received $\beta$ blockade. Among the 24 survivors (follow up 1.3-23.2 years, median 5.5 years) hypertrophy had resolved in nine (38\%) (idiopathic 8 , Noonan 1), was mild and asymptomatic in seven $(29 \%)$, and was symptomatic or severe in eight (33\%). All 10 infants presenting with septal thickness $>1.3 \mathrm{~cm}$ have persistent cardiac hypertrophy. Conclusions-Mortality in infant HCM is much lower than previously reported and resolution is more frequent. This may reflect increased detection of less severe forms in addition to the success of aggressive medical management including $\beta$ blockade.

Department of
Cardiology, Bristol
Royal Hospital for Sick
Children, Bristol
BS2 8BJ, United
Kingdom
J R Skinner
A Manzoor
A M Hayes
H S Joffe
R P Martin
Correspondence to:
Dr J R Skinner.
Accepted for publication
18 October 1996

(Heart 1997;77:229-233)

Keywords: hypertrophic cardiomyopathy; infant; Noonan syndrome

Hypertrophic cardiomyopathy (HCM) presenting in infancy has been shown to have a very high mortality, of the order of $50 \%$ within the first year. ${ }^{1}$ It has been our impression from our own practice that it is much lower. The previous paper was a series of case reports gathered from a number of large institutions in the USA and probably represented the most severe end of the spectrum of disease. This and other reports were from over a decade ago and the impact of more widespread use of echocardiography and more aggressive treatment including $\beta$ blockade has yet to be reported. No regionally based studies of infant HCM exist. We report a regionally based study of the incidence, mode of presentation, and outcome of infants with HCM over a 25 year period in the South West Region of the United Kingdom.

\section{Methods}

In 1969, a cardiac database was established in Bristol, which has recorded all children referred to the paediatric cardiology service within the South West Region of England and much of south Wales. The population base is approximately four million. Using this database, all infants presenting with HCM were identified over a 25 year period from 1969 to 1994.

Hospital notes, cardiac catheterisation data, electrocardiographs, and echocardiograms were reviewed by a single observer. The mode of presentation, treatment, any underlying conditions, and current clinical status were recorded. Infants were excluded if there was significant congenital heart disease affecting the left or right ventricular outflow tract or systemic arterial hypertension. Interventricular septal thickness and left ventricular posterior wall thickness were recorded from the long axis view in diastole by $M$ mode (if of adequate quality) or from the cross sectional images. Other features such as evidence of left or right ventricular dynamic obstruction were noted. All those included in the report had clear evidence of cardiac hypertrophy without ventricular dilatation, and had an interventricular septal thickness at least $2 \mathrm{~mm}$ greater than the 95th centile taken from normal populations. ${ }^{2-4}$ Those that underwent cardiac catheterisation alone (before echocardiography was available) had ECG changes of biventricular hypertrophy and deep $Q$ waves in the inferior leads, along with classical angiographic features.

\section{Results}

Fifty one infants with pathological cardiac hypertrophy were identified over the 25 year period. Twenty nine (57\%) had either (1) no 
apparent underlying cause or a family history of HCM (defined as "idiopathic", 21 infants), or (2) Noonan syndrome (eight infants). These 29 infants are the subject of this report.

The other 22 infants comprised 11 infants of diabetic mothers (22\%), four infants with a history of severe perinatal stress $(8 \%)$, and seven $(14 \%)$ with a variety of recognised syndromes including Friedreich's ataxia, trisomy 18, William syndrome, Beckwith syndrome, McCune-Allbright syndrome, possible SmithLemli-Opitz syndrome, and one with steroid induced HCM. These 22 infants were excluded from the subsequent analysis.

\section{INCIDENCE}

There was a marked increase in rate of reporting $\mathrm{HCM}$ over the 25 years. Comparing the first and last 12.5 years, the number of idiopathic cases rose from six to 15 and those with Noonan syndrome rose from two to six.

\section{MODE OF PRESENTATION}

Nineteen infants $(66 \%)$ presented with symptoms. These were cardiac failure in $17(59 \%)$ and cyanosis in two (7\%). The remainder presented with a heart murmur ( $n=9,30 \%)$, or screening because of a family history $(\mathbf{n}=1$, $3 \%)$.

\section{AGE AT PRESENTATION}

Sixteen infants $(55 \%)$ presented in the first week, including seven of the eight infants with Noonan syndrome. Nine $(31 \%)$ presented between 2 and 16 weeks, and four between 4 and 12 months.

\section{ECHOCARDIOGRAPHIC FEATURES AT PRESENTATION}

Echocardiograms from the time of presentation were available from all but five subjects (from the idiopathic group) in whom the diagnosis was based on cardiac catheterisation and angiographic data. Before 1982 echocardiograms were usually $M$ mode recordings alone, which were analysed along with their reported interpretation.

Values for septal thickness and ratio of septum to posterior wall were similar in the idiopathic and Noonan infants. Septal thickness in the 21 patients was $0.95 \mathrm{~cm}(0.6$ to 2.2$)$ for the idiopathic group (median (range)), and $0.91(0.8$ to 1.1$)$ for the Noonan infants. All infants had a septal thickness greater than the posterior wall. The ratio of septal to posterior wall thickness was $1 \cdot 73: 1(1 \cdot 1: 1$ to $2 \cdot 7: 1)$ in the idiopathic group and $1 \cdot 83: 1$ (1.3:1 to $2 \cdot 3: 1$ ) in the Noonan group.

There was evidence of left ventricular outflow tract obstruction in 20 infants (69\%). This included 15 from the idiopathic group, of whom nine had moderate or severe obstruction, and five from the Noonan group, of whom only one had more than mild obstruction.

\section{OUTCOME}

\section{Mortality}

There were five deaths, all within the first year, giving an overall mortality of $17 \%$. All died of progressive cardiac failure; there were no sudden deaths. Three infants who died had "idiopathic" HCM; all presented early in the series and were investigated by cardiac catheterisation. Two had severe left ventricular outflow tract obstruction in combination with right ventricular outflow obstruction. The first presented on the day of birth with cyanosis and $\frac{T}{\mathbb{D}}$ died at 3 months. The second presented at $12 \stackrel{\mathrm{N}}{7}$ weeks with cardiac failure and died at $10 \stackrel{\Rightarrow}{\equiv}$ months. Both had gross septal hypertrophy. The third infant presented at 10 weeks in severe cardiac failure with moderate left ven- 흠 tricular outflow tract obstruction and gross $\frac{0}{\sigma}$

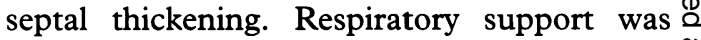
required upon arrival in hospital. He died क despite intensive support two weeks later. $\overrightarrow{0}$ None of these three infants received $\beta$ blockade.

Two infants with Noonan syndrome died. One presented on the day of birth with cardiac failure which responded to diuretic treatment. He was lost to follow up and did not receive $\beta$ i blockade, but returned at 11 months of age in $N$ severe intractable cardiac failure with evidence $ᄋ$ of gross failure to thrive. He died in intensive care within a few days. The second was $z$ preterm (32 weeks' gestation) and presented with cardiac failure requiring ventilation at $\stackrel{\rho}{\supset}$ 9 weeks of age. Cardiac catheterisation $\vec{\theta}$ showed severe left and right ventricular outflow tract obstructions $(100 \mathrm{~mm} \mathrm{Hg}$ and $70 \mathrm{~mm} \mathrm{Hg}$, respectively). She died at $7 \sum^{\circ}$ months despite $\beta$ blockade; her course was also complicated by lung disease.

Postmortem histology revealed diffuse $\stackrel{\circ}{\varnothing}$ myocardial disarray in every case. Persistence of cardiac hypertrophy and presence of $\frac{3}{3}$
symptoms

At the time of writing the median follow up of

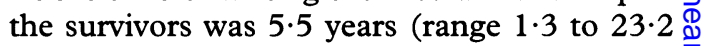
years). Cardiac hypertrophy had resolved completely on echocardiography in nine of the 24 survivors (38\%); eight of these were among the 18 survivors of the idiopathic group (44\%) and one was one of the six survivors with $\mathrm{O}$ Noonan syndrome $(17 \%)$. Of the 15 survivors? with persistent HCM, eight (53\%) had either severe echocardiographic features of HCM, or were symptomatic upon exertion with either of dyspnoea or dizziness. None had reported syn- N copal episodes or troublesome palpitations.

\section{Idiopathic HCM}

Ten of the surviving 18 subjects from the idio- $\frac{C}{\infty}$ pathic group have persisting HCM. These $\stackrel{?}{+}$ families have undergone echocardiographic $T_{T}$ screening, but only three of these 18 have evidence of familial HCM. One of the 10 with $\stackrel{\Phi}{\stackrel{D}{\circ}}$ persisting HCM had a known family member $\Omega$ with HCM (the father). In another, screening revealed suspicious ECG changes in the father $ᄋ$ but a normal echocardiogram. All of these 10 음 infants had received and are receiving $\beta$ blockers, most at a dose of approximately $3 \mathrm{mg} / \mathrm{kg} / \underset{\rightleftharpoons}{\not}$ day of propranolol. Two infants in whom HCM resolved are particularly remarkable. One subject, now aged 3 years, presented for screening because the father had HCM. Septal 
thickness at 6 weeks was slightly increased at $0.65 \mathrm{~cm}$, but is now $0.4 \mathrm{~cm}$, and there is now no evidence of HCM. Another presented at 7 days, after an uneventful perinatal period, with mild cardiac failure, a septal thickness of $1 \cdot 20$ $\mathrm{cm}$, and systolic anterior motion of the mitral valve. At 6 months, with no $\beta$ blockade, septal thickness was normal and remains so at 5 years.

Presenting features and echocardiograms in those children with a bad cardiac outcome (persisting HCM or death) were compared with those showing complete resolution of HCM. There were no significant differences in mode or age of presentation. Septal thickness was, on average, significantly higher $(P<$ 0.001 ) among those with a bad outcome (mean $1.41 \mathrm{~cm}$, range 0.87 to 2.17 ) than among those with subsequent resolution (mean $0.84 \mathrm{~cm}$, range 0.60 to 1.25 ). The infants who only underwent cardiac catheterisation all had severe HCM and although it was not possible to estimate septal thickness accurately, review of the angiograms suggests that it was above $1.5 \mathrm{~cm}$ in all cases. This being so, all 10 infants with a presenting septal thickness above $1.3 \mathrm{~cm}$ have persisting HCM or have died, while septal thickness was less than 1.3 $\mathrm{cm}$ in all eight in whom hypertrophy resolved. However, a further three infants who presented in the first week in cardiac failure with a septal thickness of approximately $0.9 \mathrm{~cm}$ $(0.87,0.88,0.90 \mathrm{~cm}$ respectively) have persisting HCM.

Four of the 10 children with persistent HCM have exertional dyspnoea; however, there seems to be little relation between symptoms and the echocardiographic features. One 7 year old girl has an outflow tract gradient in excess of $100 \mathrm{~mm} \mathrm{Hg}$ but is currently asymptomatic.

\section{HCM with Noonan syndrome}

Five of the six survivors with Noonan syndrome have persisting HCM (all of whom are receiving $\beta$ blockade), although only two are symptomatic.

\section{INFLUENCE OF $\beta$ BLOCKADE}

Since the late 1970 s it has been our policy to give $\beta$ blockade to children with symptomatology, significant and persistent hypertrophy, or outflow tract obstruction. The potential influence on mortality, symptoms, and septal hypertrophy was reviewed.

\section{Mortality}

Three infants in the idiopathic group died; none had received $\beta$ blockade. These infants represent three of the first five patients in this series (years 1969 to 1977 ). All were symptomatic at presentation (on the day of birth, at 12 weeks, and at 10 weeks, respectively). On the other hand, all 10 of the survivors in the idiopathic group who on follow up still have evidence of HCM have received $\beta$ blockade from presentation. These survivors include the other two infants of the first five in the series (the first to receive $\beta$ blockade) as well as infants with similar severity of disease to those that died; all presented with symptoms or at least moderately severe HCM.

One baby with Noonan syndrome died at 11 months without having had $\beta$ blockade; the other was preterm and died having received $\beta$ blockade. Five of the other six survivors with persisting HCM received $\beta$ blockade. Interestingly, the only patient with Noonan syndrome and resolution of HCM has never received $\beta$ blockers; presentation was at 7 days with a murmur secondary to moderate pulmonary stenosis; septal thickness was $0.9 \mathrm{~cm}$ (average for this group) and fell to $0.7 \mathrm{~cm}$ by 9 months. This mild residual hypertrophy is considered to be secondary to the valve stenosis.

\section{Symptoms}

Three children with idiopathic HCM have had $\beta$ blockade withdrawn for a variety of reasons over the years and experienced worsening shortness of breath on exertion; this improved when the treatment was restarted.

\section{Resolution of hypertrophy}

In two of the three children mentioned above, symptomatic improvement appeared to correlate with some reduction in septal thickness. In particular an 11 month old girl with idiopathic HCM had a septal thickness of $2.2 \mathrm{~cm}$ at presentation. Off $\beta$ blockade this increased to $2.6 \mathrm{~cm}$ at 19 months. Atenolol was then begun with symptomatic improvement, and at 4 years septal thickness was $1.8 \mathrm{~cm}$. Another infant presented at 5 months with septal thickness of $1.85 \mathrm{~cm}$ and was started on $\beta$ blockade; at 4 years and 4 months it was $1.3 \mathrm{~cm}$.

However, analysis of serial data available in some of the patients shows that considerable changes can occur apparently at random over time. It was noticeable that the increase in septal thickness with time among the surviving children with Noonan syndrome continued broadly parallel to the 95 th centile of normal populations, whereas there was more variability among the idiopathic group.

\section{Discussion}

Published evidence to date has suggested that presentation with isolated cardiac hypertrophy in infancy carries a grave prognosis, half the affected infants dying in the first year of life. The present report, the first regional study of infant HCM, has shown that the majority of these infants now survive at least well into childhood. Among infants with idiopathic HCM the mortality rate was $14 \%$. It was also previously reported that presentation with cardiac failure was a particularly ominous sign; Maron et $a l^{1}$ reported that nine of 11 infants (82\%) with idiopathic HCM presenting with cardiac failure died within the first year of life despite antifailure medication and sometimes surgery. Twelve infants presented with cardiac failure in the present study, and only two of these died $(17 \%)$. No infants in the present study underwent surgery, but of the 14 infants with idiopathic HCM and symptoms of either cardiac failure or cyanosis 10 received $\beta$ block- 
ade as part of their management and all were still alive a median of 4.6 years later; the three deaths occurred among the four who did not receive $\beta$ blockade.

Not only have the majority of patients survived, but most are now asymptomatic, and in one third cardiac hypertrophy has resolved.

There are several possible reasons for the reduced mortality in this series. First, this study was region based. Institution based studies are biased to more severe cases when they originate from hospitals specialising in the condition. In a report of adult $\mathrm{HCM},{ }^{5}$ a metaanalysis of 78 studies revealed that 2483 of 3404 patients (73\%) came from only two referral institutions; $44 \%$ had moderate to severe symptoms compared to only $4 \%$ in a more typical outpatient population. A second factor may be the more liberal use of $\beta$ blockade since the earlier studies, as testified by the reduction in mortality among symptomatic cases. A third factor is the more ready availability of echocardiography and paediatric cardiac services in general, which has led to the increased detection of milder and asymptomatic cases; the first five idiopathic cases from 1969 to 1977 were all symptomatic compared to only eight of the 16 between 1980 and 1994.

It might be argued that the infants in whom cardiac hypertrophy resolved do not have true HCM at all. This could be the case if the definition of HCM is histological detection of diffuse myocardial disarray. Some may have had diffuse myocyte hypertrophy rather than disarray, ${ }^{6}$ perhaps secondary to unrecognised perinatal stress; such hypertrophy would normally resolve. We do not know if these patients have myocardial disarray, as myocardial biopsy has not been performed. They presented with similar symptomatology to those with persistent hypertrophy, and one of them has a parent with HCM, making myocardial disarray more likely.

The clinical problem is that when infants with isolated cardiac hypertrophy first present, the cardiologist is not given a histological diagnosis with which to assess prognosis, but instead is presented with clinical and echocardiographic evidence of disproportionate cardiac hypertrophy. ${ }^{7}$

Infants with HCM associated with Noonan syndrome fared less well than those in the idiopathic group-HCM resolved in only one of the eight infants, and two died. Their long term outcome is rather uncertain but the risk of sudden death in later life may be lower in this group than in the familial or idiopathic group. ${ }^{8}$ Between $20 \%$ and $25 \%$ of subjects with Noonan syndrome have HCM, ${ }^{9}$ and the myocardial disarray is identical to that seen in idiopathic HCM. ${ }^{10}$

As with earlier reports, all deaths in this series were from progressive cardiac failure; there were no cases of sudden death. Although reports of sudden death in infancy and young children with idiopathic HCM are extremely rare, ${ }^{11}$ it has been reported that diagnosis in childhood statistically increases the risk of sudden death, even when the child is asympto- matic. ${ }^{11} 12$ Annual mortality may be as high as $4.8 \%$. Such evidence is again accrued through centralised reporting to large institutions and has almost certainly -in relation to the general population today-overestimated the risk. Our patients with idiopathic HCM have not all been investigated by 24 hour ECG recording but none has experienced symptomatic arrhythmia. This study may have underestimated the risk of sudden death if some infants have died suddenly outside the regional centre (or have been stillborn $^{13}$ ) and were not reported, but the South West Region has a centralised regional pathology service which $\frac{\bar{D}}{\vec{D}}$ specialises in sudden infant death, making this $\stackrel{\mathbb{Q}}{2}$ less likely. Since the close of this study a six क month old infant died suddenly within this region and was found to have myocardial fibre disarray - the first such case ever to be identi- $\vec{\omega}$ fied in this region. The peak incidence of sudden death in HCM occurs during adolescence so it is possible this could affect the patients we studied.

The families of the patients with HCM per- N sisting into the second year have undergone 0 echocardiographic screening, although this was not done for all the families of infants who 3 died early in the series, nor have any undergone detailed genetic testing for familial HCM $\stackrel{\bigcirc}{\supset}$ markers. ${ }^{14-16}$ Only three infants were found to $\vec{\bullet}$ have evidence of familial HCM (cardiac hypertrophy has resolved in one of these). The true aetiology therefore remains unresolved in the majority of this group. Recent work has suggested that a proportion may, after detailed investigation, prove to have mitochondrial $\stackrel{\varnothing}{\varnothing}$ enzyme defects affecting cardiac muscle alone $^{15}$ that might theoretically respond to car- $\frac{9}{3}$ nitine treatment. ${ }^{17}$ None of our patients received carnitine. None of the infants had clinical evidence of a generalised disorder other than Noonan syndrome, making the presence of a major systemic metabolic abnormality ${ }^{18}$ unlikely.

$\beta$ blockers are now a central part of the treatment of HCM at all ages, but there is no certain evidence to date that they prolong life, $\mathrm{O}$ reduce septal hypertrophy, or reduce the incidence of sudden death. Risk of sudden death $\frac{7}{0}$ in high risk groups of adults with HCM is reduced by low dose amiodarone, but not by $\beta$ ज blockers ${ }^{19}{ }^{20}$ or verapamil. ${ }^{21}$ However, we have $N$ found, like others, that $\beta$ blockade often $N$ results in dramatic symptomatic and haemodynamic improvement. This observation, and the fact that infants with HCM usually suc- $\frac{\bar{D}}{\bar{D}}$ cumb from cardiac failure rather than arrhyth- $\stackrel{\infty}{?}$ mic death, lends support to the earlier 7 suggestion that drugs are at least partly responsible for the reduction in mortality over $\stackrel{\odot}{\stackrel{D}{9}}$ the last 25 years. The evidence from this paper $\triangle$ that septal thickness may decrease in the longer term due to $\beta$ blockade is of itself $\Omega$ unconvincing and requires further study; how- $\frac{0}{0}$ ever, it would seem prudent to give $\beta$ blockers at least to symptomatic patients, and probably to all those with marked septal hypertrophy and outflow tract obstruction, provided they are not contraindicated for other reasons. The optimal dose is unknown, but higher dosage 
regimens may prove to be beneficial. Symptomatic benefit has been as good if not better with calcium channel blockers such as verapamil in adults with HCM. ${ }^{22}$ These agents also are incompletely evaluated in children, but are at least a reasonable second line treatment in children when $\beta$ blockers are contraindicated.

Should the term "HCM" be reserved only for those with myocardial disarray, and "left ventricular hypertrophy" be used for secondary cardiac overgrowth, as argued by Silver and Silver? ${ }^{6}$ This division is perhaps helpful for the pathologist, but is not helpful to the clinician confronted with an infant with asymmetrical hypertrophy of unknown cause and no histology to hand. As even genetically determined types of HCM become subdivided, the margins between primary and secondary HCM are becoming blurred. ${ }^{715}$ The aetiological classification of Davies and McKenna ${ }^{14}$ may be of more pragmatic use. All varieties are termed "HCM" in recognition of their clinical similarities, but they are subdivided: "familial" HCM, now identified to numerous different chromosome sites, "genetic phenotype mimics", such as Noonan syndrome and Friedreich's ataxia, "clinical mimics" such as infants of diabetic mothers and steroid induced HCM, and "exaggerated physiological response" including stress and hypertensive responses and athletes heart. The first two groups only are thought to have widespread myocardial disarray.

This study does not give a "true" incidence of infant HCM - this would require a screening programme. Nor has it been established that HCM which has resolved will remain thus for life. Some patients may have had an unrecognised stress and responded with cardiac hypertrophy ${ }^{23}$ (and perhaps were genetically predisposed to respond in this way); others may have familial HCM which will manifest itself later in life. However, this report is more representative of the true spectrum of the condition than previous reports and shows an increasing rate of detection, a higher incidence of resolution, and a lower and probably reducing mortality.

The results suggest that resolution of idiopathic cardiac hypertrophy during childhood is unlikely if the interventricular septal thickness exceeds $1.3 \mathrm{~cm}$ in the first few months of life, whereas it is likely to resolve if it is less than $0.8 \mathrm{~cm}$. The long term significance of transient hypertrophy remains unknown, though its cause is probably multifactorial. Eventually, even within a few years, it may be possible to reach an accurate cellular or molecular diagnosis in the majority of patients at birth or upon initial detection. ${ }^{14}$ For the time being this report gives more realistic figures from which to generate a prognosis when faced with the clinical problem of an infant with hypertrophic cardiomyopathy.

The South West regional cardiac database was established and maintained by Dr Steve Jordan. We are grateful to our echocardiographer Lesley Foreman for her assistance with this project.

1 Maron BJ, Tajik AJ, Ruttenburg HD, Graham TP, Atwood $\mathrm{GF}$, Victoria BE, et al. Hypertrophic cardiomyopathy in infants: clinical features and natural history. Circulation 1982;65:7

2 Solinger R, Elbl F, Minhas K. Echocardiography in the normal neonate. Circulation 1973;48:109-18.

3 Hagan AD, Deely WJ, Sahn D, Friedman F. Echocardiographic criteria for the normal neonate. Circulation 1973;48:1221-6.

4 Henry WL, Gardin JM, Ware JH. Echocardiographic measurements in normal subjects from infancy to old age. Circulation 1980;62:1054-61.

5 Spirito P, Chiarella F, Carratino L, Berisso MZ, Bellotti P, Vecchio C. Clinical course and prognosis of hypertrophic cardiomyopathy in an outpatient population. $N$ Engl $\mathcal{F}$ Med 1989;12:749-55.

6 Silver MM, Silver MD. Left ventricular hypertrophy versus hypertrophic cardiomyopathy [letter]. $\mathcal{F}$ Pediatr 1992; 121:500-1.

7 Burch M. Hypertrophic cardiomyopathy. Arch Dis Child 1994;71:488-9.

8 Burch M, Sharland M, Shinebourne E, Smith G, Patton $M, M c K e n n a$ W. Cardiological abnormalities in Noonan syndrome: phenotypic diagnosis and echocardiographic assessment of 118 patients. F Am Coll Cardiol 1993 22:1189-92.

9 Burch M, Mann JM, Sharland M, Shinebourne EA, Patton MA, McKenna WJ. Myocardial disarray in Noonan syndrome. Br Heart f 1992;68:586-8.

10 Sharland M, Burch M, McKenna WJ, Patton MA. A clinical study of Noonan syndrome. Arch Dis Child 1992; 67:178-83.

11 Maron BJ, Cecchi F, McKenna WJ. Risk factors and stratification for sudden cardiac death in patients with hypertrophic cardiomyopathy. $B r$ Heart $\mathcal{f}$ 1994;72(suppl): trophic

12 McKenna WJ, Deanfield JE. Hypertrophic cardiomyopathy: an important cause of sudden death. Arch Dis Child thy: an important

13 Landing BH, Recalde AL, Lawrence TYK, Shankle WR. Cardiomyopathy in childhood and adult life, with emphasis on hypertrophic cardiomyopathy. Pathol Res Pract 1994;190:737-49.

14 Davies MJ, McKenna WJ. Hypertrophic cardiomyopathypathology and pathogenesis. Histopathology 1995;26: 493-500

15 Obayashi $T$, Hattori K, Sugiyama S, Tanaka M, Itoyama S, Deguchi $\mathrm{H}$, et al. Point mutations in mitochondrial DNA in patients with hypertrophic cardiomyopathy. Am Heart 1992;124:1263-9.

16 Watkins $\mathrm{H}$. Multiple disease genes cause hypertrophic cardiomyopathy. Br Heart $\mathcal{F}$ 1994;72(suppl):S4-9.

17 Matsiushi T, Hirata K, Terasawa K. Successful carnitine treatment in two siblings having lipid storage myopathy with hypertrophic cardiomyopathy. Neuropediatrics 1985 with hyp

18 Carvalo JS, Matthews EE, Leonard JV, Deanfield J. Cardiomyopathy of glycogen storage disease type 111 .

19 McKenna WJ, Oakley CM, Krikler DM, Goodwin JF. Improved survival with amiodarone in patients with hypertrophic cardiomyopathy and ventricular tachycardia. Br Heart $\mathcal{F}$ 1985;53:412-6.

20 McKenna WJ, Chetty S, Oakley CM, Goodwin JF Arrhythmia in hypertrophic cardiomyopathy: exercis and 48 hour ambulatory electrocardiographic assessmen with and without beta adrenergic blocking therapy. $A m f$ Cardiol 1980;45:1-5.

21 McKenna WJ, Stewart JT. Management of arrhythmias in hypertrophic cardiomyopathy. ACC Curr F Rev 1994 July/Aug:29-31.

22 Abelmann WH, Lorell BH. The challenge of cardiomyopathy. F Am Coll Cardiol 1989;13:1219-39.

23 Manetti A, De Simone L, Pollini I, Favilli S, Scarano E, Donzelli GP. Transient ventricular septal hypertrophy in the first year of life associated with neonatal brain injury [letter]. Pediatr Cardiol 1992;13:63-4. 